\title{
Neural Correlates of Memory Retrieval during Recognition Memory and Cued Recall
}

\author{
M. D. Rugg,* P. C. Fletcher,† K. Allan,* C. D. Frith,† R. S. J . Frackowiak,† and R. J . Dolan† \\ *Wellcome Brain Research Group, School of Psychol ogy, University of St Andrews, St Andrews, FifeKY16 9] U, United Kingdom; \\ and † Wel IcomeDepartment of Cognitive Neurol ogy, Institute of Neurol ogy, Queen Square, London WC1N 3BG, United Kingdom
}

Received February 20, 1998

\begin{abstract}
Regional brain activity, measured by $\mathrm{H}_{2}{ }^{15} \mathrm{O}$ PET, was investigated during recognition memory and wordstem cued recall of words in order to compare the neural correlates of two components of memory retrieval-effort and success-as a function of task. For each task there was a baseline and two retrieval conditions. In one retrieval condition (zero density), none of the test items corresponded to words encoded in a preceding study phase. Differences in activity between this condition and the baseline were employed to characterize the neural correlates of retrieval effort in each task. In the other retrieval condition (high density), $80 \%$ of the test items had been studied previously. Differences in brain activity between this condition and the zero-density condition were taken to represent the neural correlates of successful retrieval. The principal findings concern the right anterior prefrontal cortex, a region demonstrated previously to be active during episodic retrieval. Relative to baseline, this region showed no evidence of activation in the zero-density condition of the recognition task, but did show enhanced activity in the equivalent condition of the cued-recall task. In contrast, relative to the zerodensity condition, the high-density condition was associated with increased right prefrontal activity during recognition, but reduced activity during cued recall. It is proposed that the right prefrontal cortex supports cognitive processes that operate on information retrieved in response to a test item and that these processes contribute to the evaluation of whether the information represents an appropriate prior episode. 1998 Academic Press
\end{abstract}

\section{INTRODUCTION}

The cognitive operations supporting the retrieval of episodic memories have been divided into two broad classes, associated with retrieval "effort" (or "mode") and retrieval "success," respectively (Kapur et al., 1995; Nyberg et al., 1995; Schacter et al., 1996a; Rugg et al., 1996). Retrieval effort refers to operations engaged when an attempt is made to recover information from memory. Retrieval success refers to processes engaged when a retrieval attempt is successful and yields information about a past event.

Functional imaging studies of episodic memory retrieval have consistently reported activation-stronger and more widespread in the right hemisphere-in anterior prefrontal cortex. While activation has been described in a number of different prefrontal regions, it has been found most consistently in the vicinity of Brodmann area (BA) 10, suggesting that this region may be particularly heavily involved in episodic retrieval (for review see Fletcher et al., 1997). In most of these studies effort and success were not manipulated. The results of the few studies in which effort and success were varied are inconsistent (Kapur et al., 1995; Nyberg et al., 1995; Rugg et al., 1996, 1997; Tulving et al., 1994; Schacter et al., 1996a). Notably, whereas thefindings from a study of recognition memory (Rugg et al., 1996) suggest that neural activity in the prefrontal cortex covaries with retrieval success, the results of a study of cued recall (Schacter et al., 1996a) suggest that effort may bethe more important variable. The factors responsible for these discrepant findings are unclear, not least because they come from studies that employed not only different tasks, but also different ways of manipulating success.

In our previous study (Rugg et al ., 1996), success was manipulated by varying the proportion of old and new words in recognition memory test lists. Activity in several prefrontal regions covaried with the proportion of old words and hence with the probability of successful retrieval. Schacter et al. (1996a) contrasted conditions in which subjects recalled items that had been studied once in a "shallow" encoding task or four times in a "deep" task. The rationale was that deep encoding would promotehigh levels of uneffortful recall, whereas shallow encoding would lead to low levels of retrieval success, but high levels of retrieval effort. Recall of shallowly encoded items was associated with activation of prefrontal cortex, whereas recall of deeply studied 
items failed to activate prefrontal cortex. The interpretation of these findings is complicated because success and effort were confounded, making it impossible to assess the effects of one variable independent of the other.

In the present study neural activity associated with retrieval effort and retrieval success during recognition memory and cued recall was contrasted directly. Retrieval success was manipulated by varying the ratio of old and new items while holding study conditions constant. With this procedure, effort and success are not confounded, allowing their neural correlates to be assessed separately. We expected that the results from the recognition task would replicate those from our first study to employ this experimental design (Rugg et al., 1996). At issue is whether, when effort and success are unconfounded, the findings from cued recall resemble those obtained for recognition or those reported by Schacter et al. (1996a).

\section{MATERIALS AND METHODS}

\section{O verview}

There were three experimental conditions for each memory task: a control condition, in which there was no requirement to engage in memory retrieval, and two retrieval conditions, in which the test items served as cues for the retrieval of items presented in a preceding study list. The control tasks were similar to those employed in previous studies of recognition memory (e.g., Nyberg et al., 1995) and word-stem cued recall (e.g., Buckner et al., 1995; Schacter et al., 1996a) and were closely matched to their associated memory tasks with respect to perceptual and output demands. The two retrieval conditions differed with respect to the proportion of test items corresponding to studied words (target density). In the zero-density condition, no such items were presented in the middle part of the test list, whereas in the high-density condition, approximately $80 \%$ of the items in the middle part of the list corresponded to studied words. To disguise the density manipulation, and thereby minimize the likelihood that subjects would adjust their retrieval strategies according to their perception of the probability of successful retrieval, the initial and terminal sections of each test list consisted of a 50:50 mix of items corresponding to studied and unstudied words. Scanning was performed only during the presentation of the middle part of each list.

\section{Subjects}

The subjects were six healthy adults (one female, all right handed, mean age 36 years), with no history of neurological or psychiatric illness. All subjects gave written informed consent prior to participation. The study was approved by the local hospital ethics committee and the Administration of Radioactive Substances Advisory Committee of the UK.

\section{Stimulus Materials}

The stimuli were selected from a pool of words in which each item began with a unique 3-letter stem which was shared by at least a further 5 words. The words ranged in length between 4 and 10 letters and had a mean frequency of occurrence of 34 (SD = 97) per million (Francis and Kucera, 1982). The words were employed to construct 12 sets of study-test lists. Each set consisted of one study list, 21 words in length, and four corresponding test lists, each 34 items long. The items in two of the test lists were words, and these lists were employed for the recognition tasks; the other two lists consisted of the first 3 letters (the stem) of these words and were used for the cued-recall tasks. The first 14 and last 6 items of each test list were divided equally and randomly between old (previously studied) and new (unstudied) items (i.e., words or stems corresponding to these words). The intervening 14 items either were all new (zero-density conditions) or consisted of a random sequence of 11 old and 3 new items (highdensity conditions).

All stimuli were presented in black characters on a white background on a TV monitor suspended approximately $45 \mathrm{~cm}$ in front of the subject. The presentation rate of the study items were experimenter-paced and each item was displayed until it was replaced by its successor. Stimuli in the test lists were presented with a stimulus onset asynchrony of $3.5 \mathrm{~s}$ and a display duration of $500 \mathrm{~ms}$.

\section{Experimental Tasks}

As noted previously, subjects performed two versions of each memory task and two control tasks. In the recognition memory control task, subjects viewed a series of visually presented words and were required to repeat each word aloud as soon as it was presented on the monitor. In the cued-recall control task, subjects viewed a series of word stems and were required to respond to each one with the first word to come to mind.

Each memory task was preceded by an identically structured study phase. Subjects viewed each word of a study list as it was displayed on the TV monitor and responded with a short sentence incorporating the word. Responses were monitored by an experimenter, who also initiated the presentation of each item. At the end of each study phase, subjects were required to count back in 3's from an arbitrary number for approximately 2 min, after which the corresponding test task began. For recognition memory, the test instructions were to repeat each word aloud and state whether it was old or new. For cued recall, the instructions were to 
complete each stem with a word from thestudy list or, if a study word could not be retrieved, to complete the stem with the first word to come to mind. Subjects were required to say the completion out loud and to state whether it corresponded to an old (studied) or a new word. Thus, the response requirements for the two tasks were identical. For both tasks, the response on each trial was recorded to permit the analysis of memory performance.

The two control tasks, and each condition (zero and high density) of the two memory tasks, were administered twice, to give a total of 12 test blocks. The blocks were administered in an ABCDE FFEDCBA order, which differed for each subject. A different study-test set was employed for each block (in the case of the blocks corresponding to the control tasks, no study phase was undertaken). The allocation of the sets of study-test lists to blocks was arranged so that, across subjects, the sets were rotated through the control, zero-density, and high-density conditions of each task, thereby ensuring that across-task and across-condition comparisons were not confounded with item effects.

\section{Procedure}

After installation in the scanner, cannulation, and the completion of an attenuation scan, subjects were informed of the requirements for each task and were given practice runs with lists additional to those employed in the experiment proper. They were not informed that the relative proportions of studied and nonstudied items in the memory tasks would vary. As described below, the presentation of the test lists was coordinated with the onset of the scanning interval so that rCBF was measured only within the interval during which the target/nontarget ratio varied (i.e., between the 15th and the 29th item of each list). The interval between successive scans was approximately $10 \mathrm{~min}$. This interval was filled either by a study phase or, when the next test block involved a control task, by rest and conversation with an experimenter.

\section{PET Scanning}

PET scans were obtained using a Siemens/CPS ECAT EXACT HR + (Model 962) PET camera operating in 3D acquisition mode. For each scan, subjects received a 20-s intravenous bolus of $\mathrm{H}_{2}{ }^{15} \mathrm{O}$ through a forearm cannula at a concentration of $55 \mathrm{MBq} \mathrm{ml}^{-1}$ and a flow rate of $10 \mathrm{ml} \mathrm{min-1}$. With this method, each scan provides an image of rCBF integrated over a period of $90 \mathrm{~s}$ from when the tracer first enters the cerebral circulation. The first $30 \mathrm{~s}$ of this period is concurrent with the rise of radioactivity in the head and forms the window in which the experimental manipulation modifies the distribution of cerebral blood flow (Silbersweig et al., 1993). The onset of theinfusion of the $\mathrm{H}_{2}{ }^{15} \mathrm{O}$ bolus was timed so that rCBF was measured during the period between the presentation of the 15th and the 29th item of each test list.

\section{PET Data Analysis}

The attenuation-corrected PET scans were analyzed using SPM 96 (Well come Department of Cognitive Neurology, London, UK; F riston et al., 1995). The scans from each subject were realigned to correct for head movement, transformed into a standard space (Talairach and Tournoux, 1988), and smoothed using an isotropic Gaussian kernel (full-width half maximum $10 \mathrm{~mm}$ ). Condition, subject, and covariate effects were estimated for each voxel according to the general linear model, as implemented by SPM96. The specific contrasts employed to analyze the data, and their rationale, are described under Results.

\section{RESULTS}

\section{Behavioral Data}

At the end of the experimental session each subject was questioned about whether they noticed any differences between the test lists of the memory tasks. Four subjects reported that they failed to notice any differences in the structure of the lists. One subject reported that "sometimes there didn't seem to be many old words," and the remaining subject reported that there were "not consistent numbers" of old and new items.

Performance in each condition of the two tasks is summarized in Table 1 , where it can be seen that accuracy was lower in the cued-recall task than in the recognition task, but that there was little sign of an effect of target density in either task. N ote that for the cued-recall task, responses were scored as correct only when a stem was both completed with a study word and correctly classified as old (the proportion of trials in which correct completions were misclassified as unstudied was negligible), and a false alarm was recorded whenever a stem was completed with an unstudied word and misclassified as old, irrespective of whether the stem bel onged to a studied or an unstudied item.

ANOVA (factors of task and condition) on the sensitivity index pHit-pFalseAlarm showed that there

\section{TABLE 1}

Percentage (and SD) Hit and False Alarm Rates in Each Task According to Target Density

\begin{tabular}{|c|c|c|c|c|}
\hline & \multicolumn{2}{|c|}{ Cued recall } & \multicolumn{2}{|c|}{ Recognition } \\
\hline & Zero & High & Zero & High \\
\hline Hits & $49.2(16.2)$ & $51.3(4.1)$ & $96.6(4.1)$ & $94.9(6.6)$ \\
\hline False alarms & $6.3(3.3)$ & $5.7(2.4)$ & $2.4(2.0)$ & $2.6(3.1)$ \\
\hline
\end{tabular}


was a significant effect of task ( $F$ 1, $5=92.58$, $\mathrm{P}<0.001)$, but no sign of a condition effect or a task $X$ condition interaction (both F's $<1$ ). ANOVA of the false alarm rates alone revealed a significant effect for task ( $\mathrm{F} 1,5=9.78, \mathrm{P}<0.05)$, but no condition or task $\times$ condition effect. These findings indicate that subjects were more accurate in recognizing old words than they were in recalling them and that they were more likely to misclassify an unstudied stem completion than they were an unstudied word. The absence of an effect of target density suggests that subjects did not adjust their retrieval strategies according to the likelihood of retrieval success, for example, by expending less retrieval effort in the zero-density condition or adjusting their criterion for judging an item as old. This last conclusion is strengthened by an analysis of performance on the final six items of each test list, when the old:new ratio reverted to 50:50 and differential retrieval strategies that developed during the preceding part of the test sequences, when density varied, would likely be most evident. For both tasks, mean hit rates on the terminal section did not vary with target density ( $40 \%$ for both conditions of the cued-recall task, $94 \%$ for the two conditions of the recognition task), and false alarm rates were affected only minimally. One subject false alarmed once in one of the zero-density runs of the cued-recall task, and four subjects each made one false alarm in one of the high-density runs of the task. For recognition, one subject made one false alarm in one of the zero-density runs, and no false alarms at all were committed in the high-density runs.

\section{PET Data}

ThePET data were subjected to two sets of preexperimentally determined comparisons. The first set was designed to identify and compare activations associated with engagement in each of the memory tasks in the absence of successful retrieval. The second set was designed to investigate the effects of varying target density.

\section{Task Engagement}

Pairwise contrasts between the zero-density and the control conditions of each task were employed to identify regions sensitive to task engagement. To determine whether any of these regions were more sensitive to engagement in one task than the other, task $\times$ condition interaction effects were also computed, and the resulting statistical parametric maps inspected for regions that overlapped with those identified by the aforementioned pairwise comparisons. Unless otherwise noted, the statistical threshold for all of these comparisons was $\mathrm{P}<0.001$, uncorrected for multiple comparisons.

The results of the pairwise comparisons are given in
Table 2. For the recognition task, the zero vs control contrast revealed bilateral activation of the cerebellum, but no other significant effects. For cued recall, the equivalent contrast revealed reliable activation of a number of regions, including the prefrontal cortex bilaterally (Fig. 1a), left parietal cortex, and the right cerebellum. The interaction testing for larger engagement effects in the recognition task than in the cuedrecall task [(zero vs control recognition) - (zero vs control cued recall)] revealed no reliable effects. The complementary analysis revealed that the effects of task engagement were significantly greater for cued recall than for recognition in the vicinity of two of the regions identified by thezero density vs control comparison of the cued-recall task (see Fig. 1b). These regions were the left anterior prefrontal cortex $(x, y, z=-22$, 44,$10 ; Z=3.17$ ) and left lateral parietal cortex $(x, y, z,=-46,-36,48 ; z=3.29)$. In addition, there was a trend for the effects of task engagement to be greater for the cued-recall task than for recognition in the right anterior prefrontal cortex $(x, y, z=34,46,4$; $Z=2.88 ; P<0.002$ )

\section{Density Effects}

Regions in which activity increased with target density in each task were identified by pairwise contrasts between the high- and the zero-density conditions, and the subsets of these regions in which density-sensitive activity varied according to task were identified by the analysis of task $\times$ condition interaction effects. In addition, pairwise contrasts were performed for each task to identify regions exhibiting greater activity in the low-density condition. These contrasts were motivated by the finding of Schacter et al. (1996b) that activity in the left prefrontal cortex was greater for the recall of shallowly studied items, when retrieval suc-

\section{TABLE 2}

$x, y$, and z Coordinates (Talairach and Tournoux, 1988), z Values, Corresponding Brain Regions, and ApproximateBrodmann Areas (BAs) of Peak Increases in rCBF for the Contrasts between the Zero-Density Condition of Each Task and Its Corresponding Control Condition

\begin{tabular}{|c|c|c|c|c|}
\hline & Coordinates & Z & Region & BA \\
\hline Recognition & $\begin{array}{c}20,-88,-36 \\
-30,-40,-30\end{array}$ & $\begin{array}{l}3.45 \\
3.14\end{array}$ & $\begin{array}{l}\text { Right cerebellum } \\
\text { Left cerebellum }\end{array}$ & \\
\hline \multirow[t]{8}{*}{ Cued recall } & $36,50,4$ & 4.01 & Right anterior prefrontal & 10 \\
\hline & $-22,46,10$ & 3.91 & Left anterior prefrontal & 10 \\
\hline & $-44,36,0$ & 3.30 & Left dorsolateral prefrontal & $45 / 47$ \\
\hline & $56,-56,52$ & 3.14 & Right lateral parietal & 40 \\
\hline & $-36,-46,46$ & 4.00 & Left lateral parietal & $7 / 40$ \\
\hline & $-8,-78,36$ & 3.21 & Left medial occipital & $18 / 19$ \\
\hline & $-32,-98,4$ & 3.68 & Left lateral occipital & 18 \\
\hline & $14,-80,-42$ & 3.83 & Right cerebellum & \\
\hline
\end{tabular}




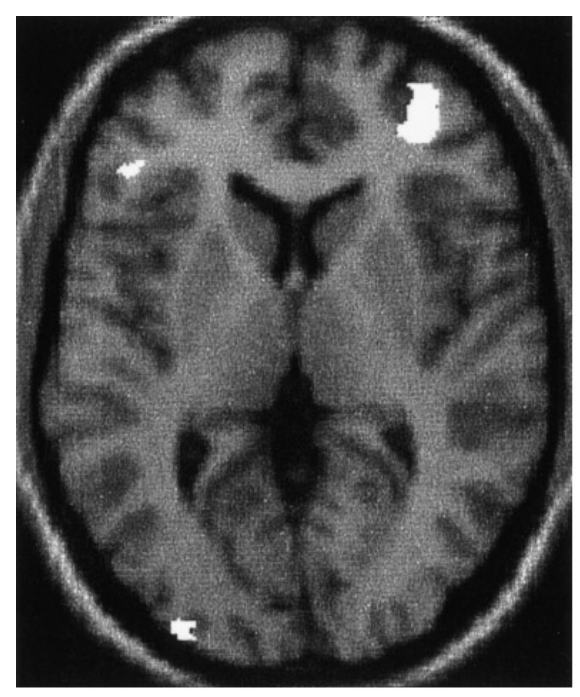

a

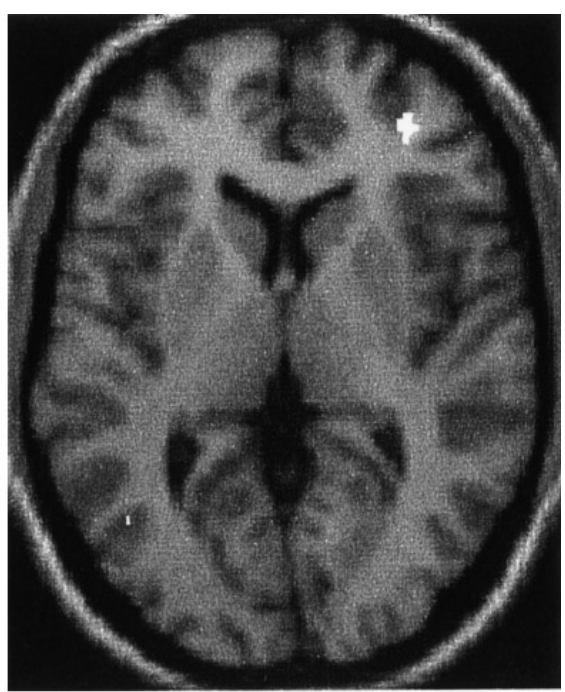

b

FIG. 1. Statistical parametric maps (threshold $P<0.01)$ superimposed onto transverse sections $(z=0)$ of a magnetic resonance brain image which has been transformed into standard stereotactic space (Talairach and Tournoux, 1988). (a) The effects of engagement in the cued-recall task (zero density vs control) showing bilateral prefrontal activations. (b) The right prefrontal locus at which engagement effects were greater for the cued recall than for the recognition task (task $\times$ condition interaction).

cess was relatively low, than it was for the easier condition involving the recall of deeply studied words.

To increase their sensitivity without creating an unacceptable risk of type I error, the contrasts testing for density effects were "masked" by the contrast between all of the memory conditions and the two control conditions, restricting the search space for density effects to the regions activated by the two memory tasks relative to their respective control conditions. This procedure is analogous to the one adopted in our previous study of target density effects (Rugg et al., 1996) and provides a hypothesis-driven alternative to methods that control type I error for an entire population of voxels. The masking contrast was thresholded at $\mathrm{P}<0.01(\mathrm{Z}=2.31)$, and the voxels within the mask where the contrasts testing for density effects also exceeded the $\mathrm{P}<0.01$ significance level were considered sensitive to the factor of target density. The conjoint probability of a voxel surviving both sets of contrasts is considerably lower than that for either contrast al one (approximately $\mathrm{P}<0.0001$ ). Note, however, that the $Z$ values reported in Table 1 and below correspond to the results of the specific contrasts testing for the effects of density.

The results of the specific contrasts between the high- and the low-density conditions are given for each task in Table 3. For the recognition task, high target density was associated with greater activation of right prefrontal cortex, the medial parietal cortex, and lateral parietal cortex bilaterally (Fig. 2). For the cuedrecall task, the analogous contrast revealed activation in the vicinity of the medial parietal cortex (Fig. 3) and, to a lesser extent, the left lateral parietal cortex. There was, however, no evidence of greater activity in the high-density condition in any part of the prefrontal cortex.

No regions were more active during the zero-density than during the high-density condition of the recognition task. In the case of cued recall, greater activity in the zerodensity condition was found in several regions, including the left and right prefrontal cortex (see Table 3).

The analyses of the task $\times$ condition interactions revealed that density effects in the recognition task exceeded those in the cued-recall task in several of the regions identified by the high vs zero contrast for the recognition task (see Fig. 4). These regions included right anterior prefrontal cortex $(x, y, z=32,44,18$; $Z=2.73$ ), posterior and anterior regions of the left lateral parietal cortex (posterior: $x, y, z=-34,-76$, $-38 ; z=3.66$; anterior: $x, y, z=-34,-46,38 ; z=2.98)$, and right lateral parietal cortex $(x, y, z=34,-64,54$; $Z=2.88)$. The complementary interaction, testing for greater density effects in cued recall, revealed a trend for a greater effect in the left medial parietal cortex ( $x$, $y, z=-4,-68,46 ; z=1.85)$.

\section{Task Effects at High Target Density}

Because of its theoretical significance (see Discussion), an additional masked comparison was performed to determine whether the right prefrontal cortex was differentially active in the high-density conditions of the two tasks. As shown in Fig. 5, the comparison revealed higher activity in this region during recogni- 
TABLE 3

$x, y$, and z Coordinates, Z Values, Corresponding Brain Regions, and Approximate Brodmann Areas (BAs) of Peak Increases in rCBF for the Contrasts between the High-Density and Zero-Density Conditions of Each Memory Task

\begin{tabular}{|c|c|c|c|c|}
\hline & Coordinates & Z & Region & BA \\
\hline \multicolumn{5}{|l|}{ Recognition } \\
\hline & $32,50,18$ & 2.73 & Right anterior prefrontal & 10 \\
\hline & $-34,-50,44$ & 2.31 & Left lateral parietal & $7 / 40$ \\
\hline & $34,-64,54$ & 3.28 & Right lateral parietal & 7 \\
\hline & $-36,-80,40$ & 4.09 & Left lateral occipital & 19 \\
\hline & $-2,-74,36$ & 2.66 & Left medial parietal & $7 / 31$ \\
\hline \multicolumn{5}{|l|}{ Cued recall } \\
\hline \multicolumn{5}{|l|}{ High vs zero } \\
\hline & $8,-72,22$ & $\begin{array}{l}3.31 \\
3.25\end{array}$ & Right medial occipital/parietal & $18 / 31$ \\
\hline & $-36,-66,58$ & 2.42 & Left lateral parietal & 7 \\
\hline \multicolumn{5}{|l|}{ Zero vs high } \\
\hline & $-22,46,12$ & 3.09 & Left anterior prefrontal & 10 \\
\hline & $22,44,-6$ & 2.41 & Right anterior prefrontal & 10 \\
\hline & $12,-32,22$ & 3.27 & Right posterior cingulate & 31 \\
\hline & $-36,-46,38$ & 2.49 & Left lateral parietal & 40 \\
\hline & $14,-82,-42$ & 3.44 & Right cerebellum & \\
\hline
\end{tabular}

Note All contrasts were masked by the orthogonal contrast between all memory and all control conditions (see text).

tion than during cued recall (peaks at $x, y, z=24,42$, $-8 ; z=4.47 ; x, y, z=38,44,4 ; z=3.42)$.

\section{Summary of Results}

The most important findings concern the levels of activity of right anterior prefrontal cortex in the different experimental conditions. These areillustrated quantitatively in Fig. 6 for the voxel in the vicinity of BA 10 that showed maximum sensitivity to the manipulation of target density in the recognition task. As is evident from the figure, rCBF differed little between the control and the zero-density conditions of the recognition task, but was markedly elevated during the high-density conditions of this task. By contrast, the greatest difference in rCBF for this voxel in the cued-recall task was between the control and the zero-density conditions.

\section{DISCUSSION}

The present findings converge with those from a previous electrophysiological study (Allan and Rugg, 1997) to suggest that, despite a shared reliance on the hippocampal formation and its associated structures (Squire, 1992), recognition memory and word-stem cued recall nonetheless have distinct neural correlates. In particular, there was a striking dissociation between the two tasks in the sensitivity of the prefrontal cortex to the factors of task engagement and target density. The dissociation is consistent with findings from previous studies of recognition (Rugg et al., 1996) and cued recall (Schacter et al., 1996a) and indicates that the disparate results of these studies cannot be attributed to the different methods by which retrieval effort and success were manipulated. The dissociation highlights the importance of separating the effects of task engagement and successful retrieval in neuroimaging studies and calls into question the utility of comparisons between memory tasks when these effects are confounded (e.g., Cabeza et al., 1997).

\section{Right Prefrontal Cortex}

The finding that the zero-density recognition condition failed to activate right prefrontal cortex is contrary tothe results of Nyberg et al. (1995). Thereason for this contradiction may lie with the proportion of false alarms committed in each study, which was substantially higher in Nyberg et al. (1995) than in the present case ( $17 \%$ vs $2.5 \%)$. If the right prefrontal cortex is activated when a test item elicits retrieval irrespective of whether the retrieved information is veridical (Schacter et al., 1996c), the findings of Nyberg et al . (1995) can be interpreted without recourse to the notions of re trieval effort or attempt.

The finding that the right prefrontal cortex was sensitive to the density manipulation in the recognition task replicates our previous result (Rugg et al., 1996; see Kapur et al., 1995 and Nyberg et al., 1995 for contradictory findings and Fletcher et al., 1997 for discussion). Together with the failure to find evidence of prefrontal activation in the zero-density condition, this finding adds weight to the proposal, advanced on the basis of both hemodynamic (Rugg et al., 1996) and electrophysiological (Allan et al., 1998; Wilding and Rugg, 1996) data, that right prefrontal cortex supports 

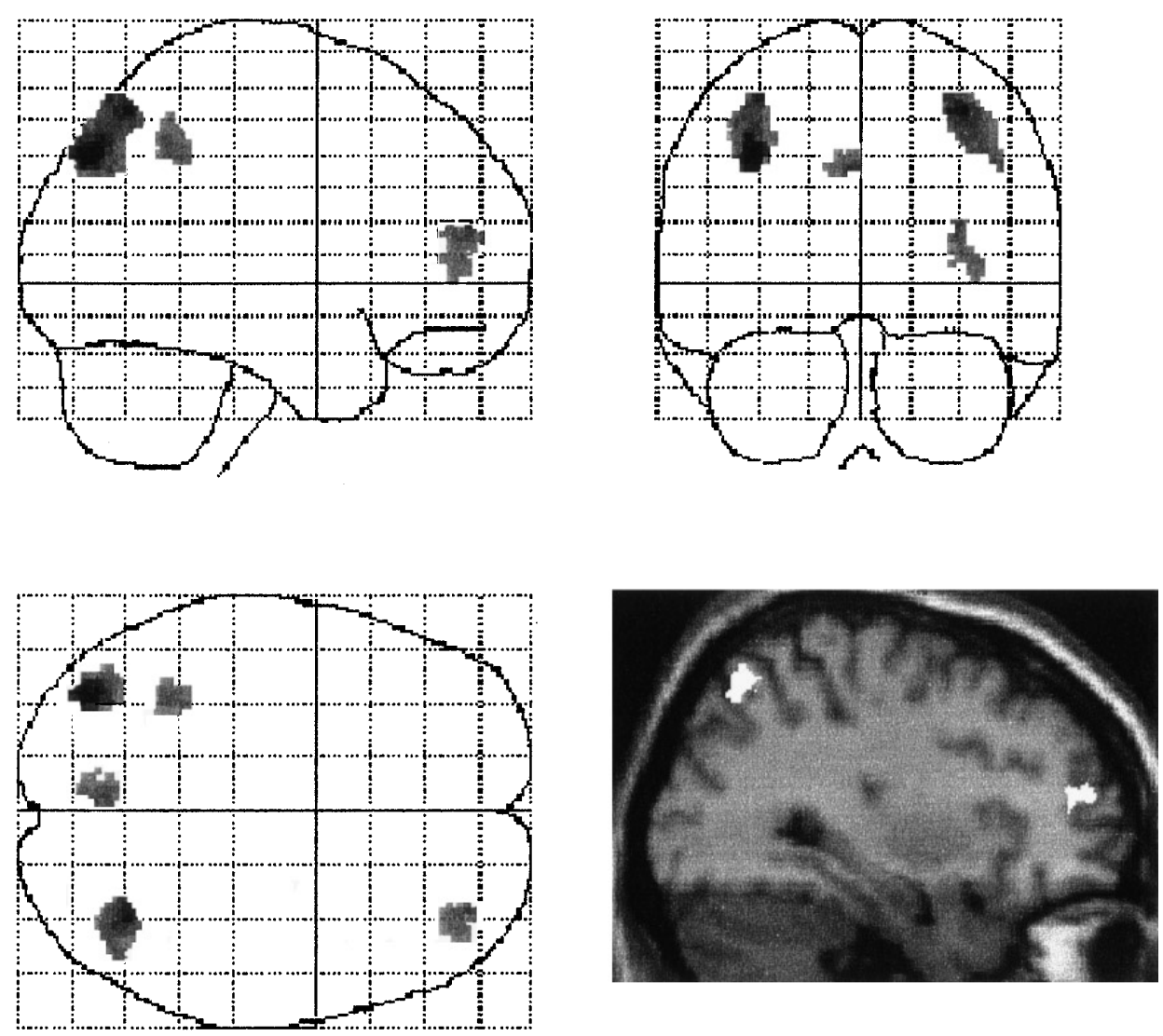

FIG. 2. Maximum intensity projection (threshold $P<0.05$ ), and its projection onto a saggital section ( $x=32$ ) of a standard magnetic resonance image, of the outcome of the contrast between the high- and the zero-density conditions of the recognition task.

processes that act on the products of retrieval, rather than processes engaged during attempted retrieval.

The findings from the recognition task also bear comparison with those from two recent studies of recognition memory employing fMRI (Buckner et al., 1998a,b). In the first of these studies (Buckner et al., 1998a) a blocked experimental design was employed in which recognition memory judgments were required on test words that had been studied under deep or shallow encoding conditions. On the basis of the finding that the deeply studied items attracted both higher hit rates and shorter RTs, Buckner et al. (1998a) concluded that the former items elicited higher levels of retrieval success than did the latter items, and for the expenditure of less effort. In keeping with the retrieval success hypothesis, BOLD signal from right anterior prefrontal cortex (BA10) was reliably greater during recognition of the deeply studied words than during recognition of the shallowly studied items.

In the second study, Buckner et al. (1998b) employed an event-related $\mathrm{fMRI}$ design in order to assess the patterns of activation associated with studied and unstudied test words on a trial-by-trial basis. Relative to a fixation-only prestimulus baseline, both classes of word gave rise to reliable hemodynamic responses in a variety of regions, including anterior right prefrontal cortex. The absence of a suitable control condition (e.g., the presentation of words in a task placing no demands on episodic retrieval) makes these findings difficult to interpret, however, as it is not clear which responses were associated specifically with processes engaged during recognition memory and which reflected more general aspects of word processing. This problem does not arise for the direct contrast of the responses el icited by studied and unstudied items. Contrary to the prediction of the retrieval success hypothesis, this contrast failed to demonstrate that the right prefrontal cortex was more active for studied than for unstudied words. The significance of this finding is uncertain, however, as reliable differences in the responses elicited by studied and unstudied words could not be detected in any brain region and not merely in the right prefrontal cortex. Since it is safe to assume that activity some where in the brain discriminated the two classes of test word, the failure to find any evidence of such differential activity calls into question the power of the study and leaves open the possibility that the null findings for the right prefrontal cortex reflect type II error. Thus, the findings of Buckner et al. (1998b) do not demand revision of the proposal that the right prefrontal cortex 


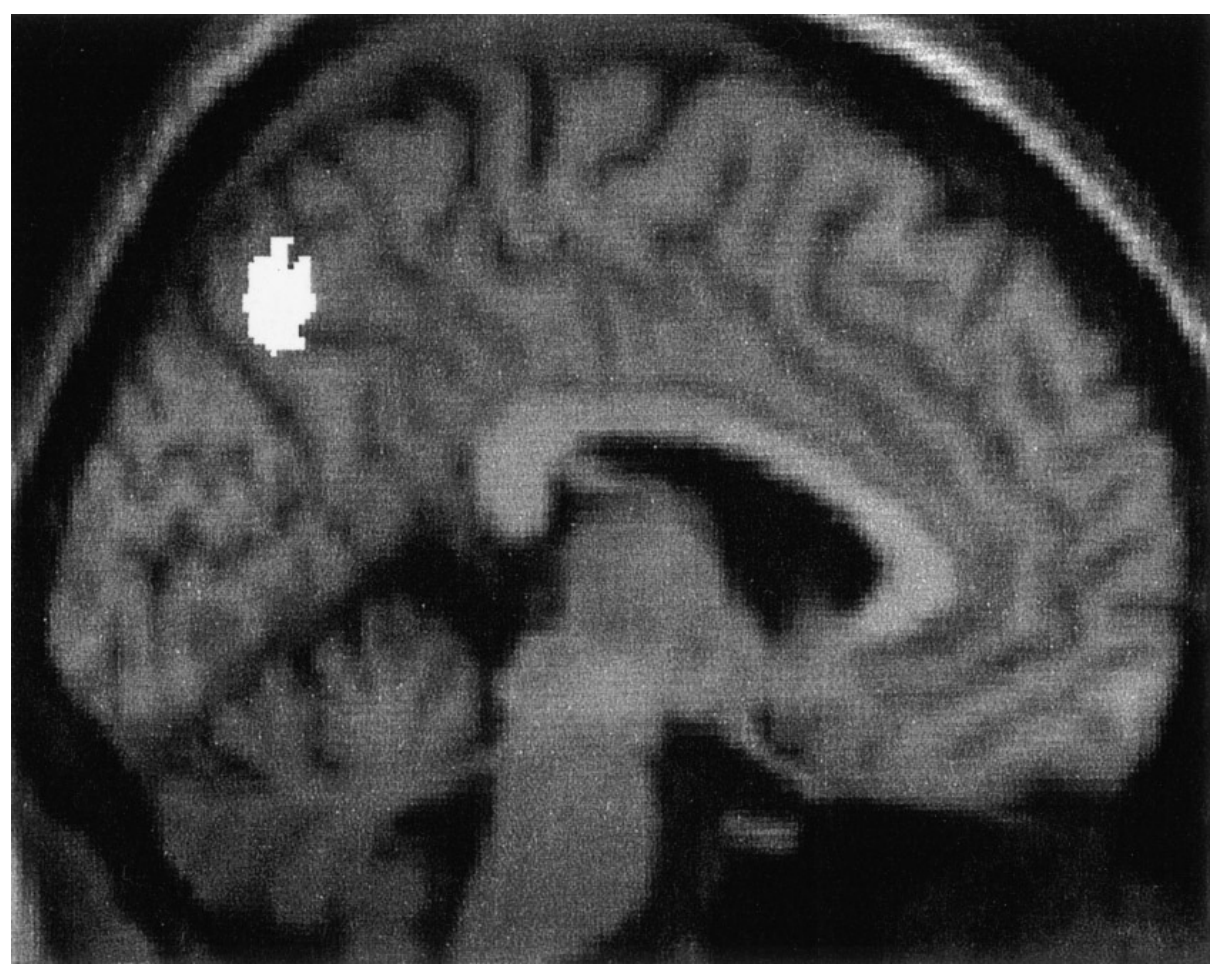

FIG. 3. Statistical parametric map (threshold $P<0.05$ ) superimposed onto a saggital section of a standard magnetic resonance image $(x=-4)$ showing the outcome of the contrast between the high- and the low-density conditions of the cued-recall task.

supports processes that act on the products of retrieval. F urthermore, the findings from Buckner (1998a) are consistent with the proposal.

The proposal is challenged, however, by the results from the present cued-recall task. In this task, engagement in the zero-density condition was associated with activation of the right prefrontal cortex, and right prefrontal activity was lower in the high-density than in the low-density recall condition.

How can the findings from the two tasks be reconciled? One approach is to view the findings from the recognition task as reflections not of the effects of successful retrieval, but of the differential allocation of effort during the zero-density and high-density conditions. By this argument, subjects detected whether test sequences came from the zero-density or the highdensity condition and adjusted their effort accordingly. This strategy was unavailable in the more difficult cued-recall task, where the probability of successful retrieval was too low to allow subjects to discriminate reliably between the two density conditions. Thus, unlike in the recognition task, effort in the recall task was allocated without regard to density. In fact, more effort was expended in the zero-density condition, when no stem gave rise to successful retrieval, than in the high-density condition, when the effortful processing of approximately $50 \%$ of the stems was terminated by successful retrieval. Thus, this interpretation accounts for the lower levels of right prefrontal activity in the high-density recall condition and for the task $\times$ density interaction illustrated in Fig. 4.

The foregoing explanation is consistent with the proposal that the right prefrontal cortex supports processes engaged by retrieval effort, but it cannot account for two aspects of the present findings. First, there is little evidence, either in the patterns of performance or from subject debriefings, to support the assumption that subjects adjusted their retrieval strategies in the recognition task according to target density (as noted by a referee, it is of course possible such adjustments did occur, but that they were neither reportable nor reflected in performance). Second, and more compelling, the direct contrast between the high-density conditions of each task revealed greater right prefrontal activity in the recognition task. Unless one is willing to argue that high-density recognition required greater effort than high-density cued recall, despite the fact that the latter was clearly the more difficult task, it is hard to reconcile this finding with the idea that prefrontal activity is associated exclusively with retrieval effort.

An alternative account of the present findings starts from the assumption that right prefrontal activation does indeed signify the engagement of processes that operate on the products of episodic retrieval, as suggested above. These processes include prefrontally mediated "verification" or "checking" operations (Koriat 

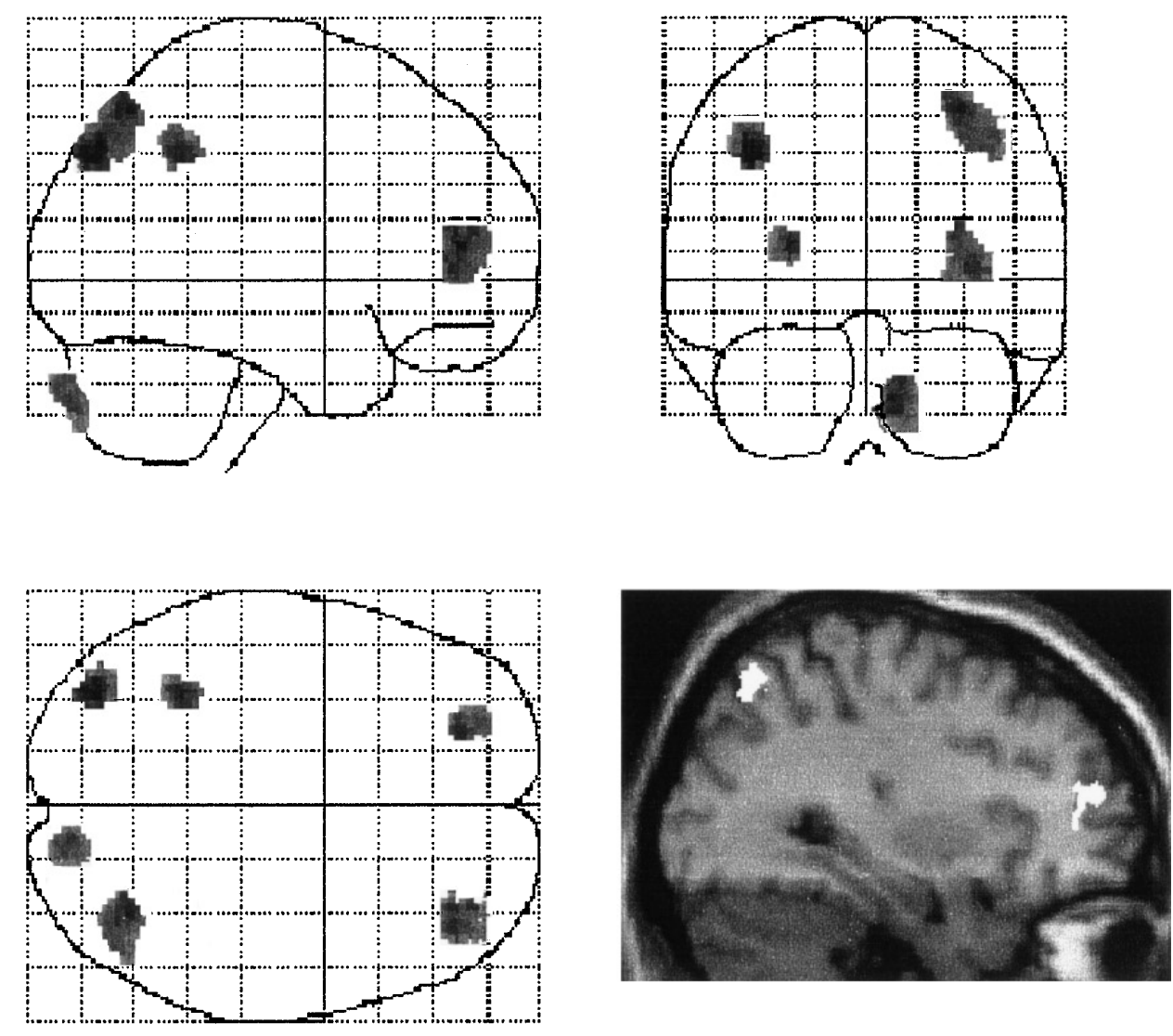

FIG. 4. Maximum intensity projection (threshold $P<0.05$ ), and its projection onto a saggital section ( $x=32$ ) of a standard magnetic resonance image, of the task $\times$ density interaction, illustrating regions where density effects are greater in the recognition task.

and Goldsmith, 1996) held, on the basis of neuropsychological findings (Shallice, 1988; Schacter et al., 1996b), to be mediated by the prefrontal cortex. The account further assumes, however, that these processes can also act on information retrieved from semantic memory; what matters is not the memory system from which information is retrieved, but that the information is evaluated with regard to whether it belongs to a specific past episode (cf. Fletcher et al., 1995a). During the zero-density condition of the cued-recall task these "postretrieval" processes are engaged because partial cues such as word stems encourage subjects iteratively to generate candidate study items, each of which is subjected to the verification and checking operations noted above. By contrast, the "copy" cues of the recognition task neither require nor permit the generation of multiple candidates for subsequent checking. In this task, therefore, postretrieval processing is confined largely to the situation-thehigh-density condition-in which significant amounts of information are retrieved in response to the test items.

The foregoing explanation accounts quite well for the current and previous findings, although further work is needed to establish its superiority over the competing effort-based account. An important feature of the expla- nation is the way in which, in accounting for the role of the right prefrontal cortex in memory retrieval, it blurs the distinction between effort and success; the operations set in train when a test item elicits successful (that is, veridical) retrieval are held also to be engaged when information retrieved or generated in response to a cue is not accepted as belonging to a "target" study episode. Furthermore, these operations are considered to require the allocation of attentional resources and hence cognitive effort. Thus, despite its heuristic value, the distinction between retrieval effort and retrieval success may ultimately be unhelpful in understanding the role of the right prefrontal cortex in memory retrieval.

\section{Left Prefrontal C ortex}

Two distinct left prefrontal regions varied in their activity during cued recall. Activation of the more anterior region (BA 10) has been described previously in studies of both cued recall (Buckner et al., 1995) and recognition (Kapur et al., 1995; Rugg et al., 1996). Despite the failure to find it in the present recognition task, it seems unlikely that activity in left BA 10 reflects processes specific to cued recall. 


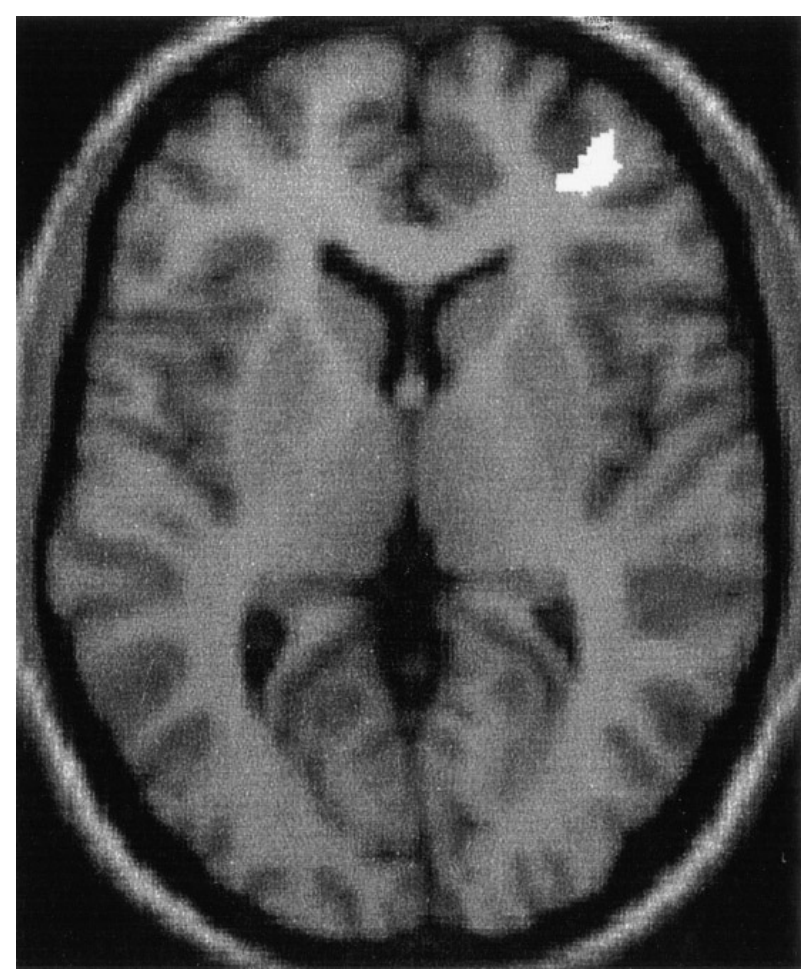

FIG. 5. Statistical parametric map (threshold $P<0.05$ ) superimposed onto a transverse section of a standard magnetic resonance brain image $(z=4)$ to illustrate the greater right prefrontal activation in the high-density condition of the recognition task than in the cued-recall task.
The locus of the left dorsolateral activation (BA 45/47) overlaps with regions that have previously been found to be activated when words are generated either overtly or covertly in response to a variety of different cues (for a review see Buckner and Petersen, 1996). Relative to the control task, when only a single word was generated on each trial, the cued recall task necessitated the covert generation of multiple re sponses to each cue (see above). Thus, the increased left dorsolateral activity observed during recall likely reflects the greater demands made by this task upon processes subserving word generation. Thefinding that activity in this region was lower in the high-density than in the zero-density condition is in keeping with this account. In the latter condition every item would have led to the generation of multiple completions. This was not the case in the high-density condition, when generation of candidate completions would have been cut short on the $50 \%$ or so of trials in which a study word was successfully retrieved.

\section{Parietal Cortex}

The high-density conditions of both tasks were associated with enhanced activity in posterior medial parietal cortex (precuneus). Although the functional role of this region in memory retrieval is obscure (cf. Fletcher et al., 1995b; Buckner et al., 1996), the present findings suggest it is sensitive to retrieval success. The findings further suggest that this sensitivity is higher for cued recall than for recognition. Despite the fact that the probability of successful retrieval was lower in the recall task, the effects of the density manipulation were

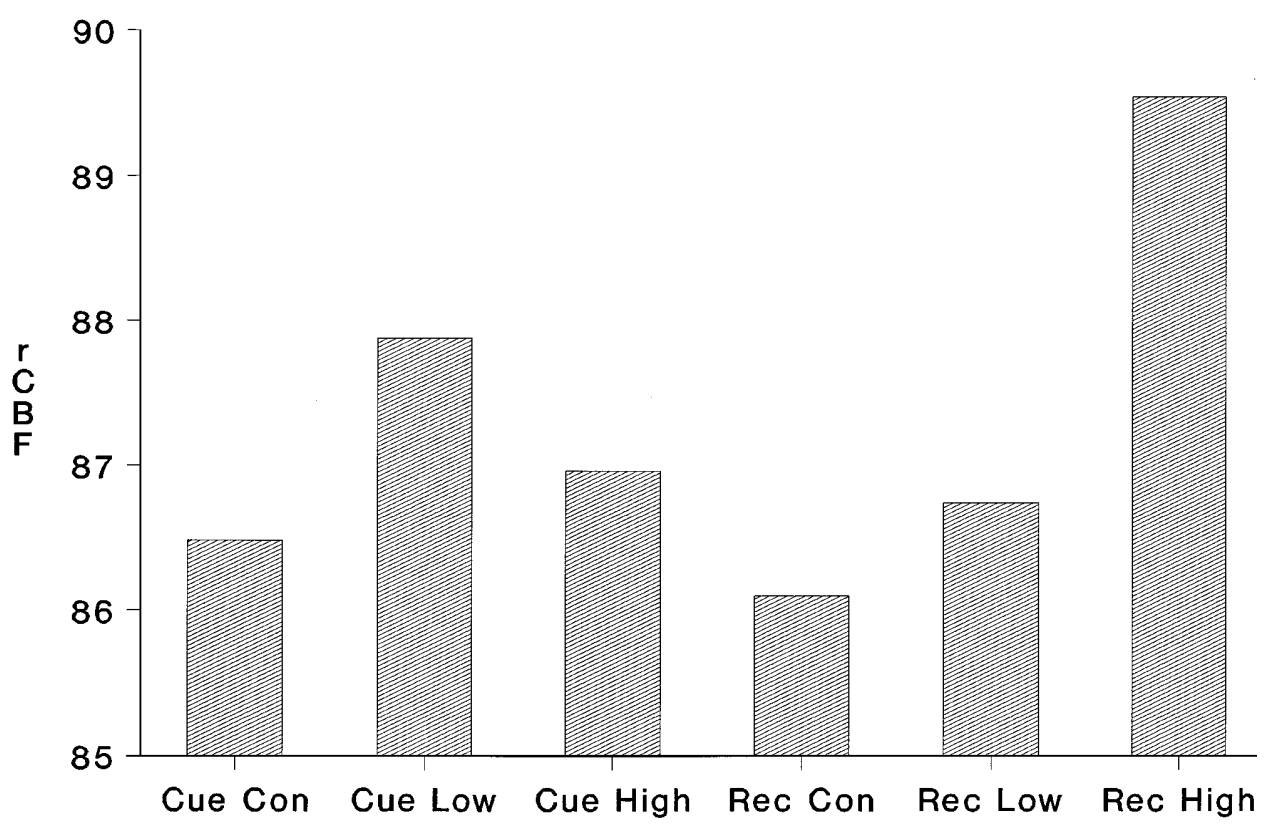

FIG. 6. Mean rCBF values (arbitrary units, adjusted for global flow) in each experimental condition from a voxel in right anterior prefrontal cortex $(x, y, z=32,50,18)$. 
no smaller than those in the recognition task; on the contrary, there was a trend in the opposite direction. Whether this finding reflects a tendency for information retrieved during cued recall to be more likely to be visually based (cf. Fletcher et al., 1995b) is an interesting question for future research.

Lateral parietal cortex was also sensitive to retrieval success, but, unlike the medial parietal region, this effect was confined largely to recognition. While this task dissociation suggests that the roles of medial and lateral parietal cortex in memory retrieval are functionally distinct, it offers little guidance as to what those roles might be.

\section{H ippocampal Formation}

The present findings differ from those reported by Schacter et al. (1996a; see also Squire et al., 1992) in one important respect. Relative to both baseline and "Iow-recall" conditions, Schacter et al. found that their high-recall condition was associated with activation in the vicinity of the hippocampal formation. These findings were interpreted as evidence for the role of the hippocampus in the recollection of study items during cued recall. In the present experiment, however, there was no evidence for greater involvement of the hippocampal region during the high-density cued-recall condition, regardless of whether this condition was contrasted with the control or the zero-density condition. A possible explanation for this disparity between the previous and the present findings lies in the levels of performance achieved in the critical conditions. In the high-recall condition of Schacter et al. (1996) subjects recalled a mean of $79 \%$ of studied words, whereas in the present experiment recall in the high-density condition averaged only about $50 \%$. Thus, the probability of successful recollection was considerably higher in the previous study, providing a better opportunity to detect recollection-related effects. That said, it should be noted that in two other studies of cued recall, in both of which performance was again substantially higher than in the present study (recall rates of $73 \%$ and $62 \%$, respectively), Buckner et al. (1995) also failed to find hippocampal activation relative to the same control condition as that employed here. Thus, the exact conditions under which successful word-stem cued recall is associated with hippocampal activation remain unclear.

The failure to find retrieval-related hippocampal activation in the recognition task of the present experiment is consistent with several previous studies of recognition memory for words (for review see Fletcher et al., 1997). This negative result is all the more striking in light of the very high performance levels achieved in the high-density condition. A possible reason for the absence of hippocampal activation in this condition lies with the fact that the basel ine conditions against which it was contrasted consisted of words that were novel to the experiment. As noted by Rugg et al. (1997; see also Fletcher et al., 1997), such items likely engender extensive encoding-related hippocampal activity, the effect of which would be to cancel any retrievalrelated activity associated with successful recognition. Consistent with this suggestion, Rugg et al. (1997; see also Dolan and Fletcher, 1997) found that recognition test lists comprising richly encoded, highly memorable items were associated with el evated hippocampal activity when contrasted with lists containing words that had been subject to shallow encoding and which, therefore, were much less memorable. Rugg et al. argued that although these latter items failed to elicit successful episodic retrieval, they were nonetheless sufficiently familiar to forestall further encoding and hence served as a better baseline against which to assess retrieval-related hippocampal activity than did words new to the experiment. The present findings are consistent with these proposals, but provide no further evidence in their favor.

\section{Concluding Comments}

The findings from the present study reconcile two seemingly conflicting sets of results (Schacter et al., 1996a vs Rugg et al., 1996) by demonstrating that activity in the right prefrontal cortex during episodic retrieval is task dependent. To account for these findings it proved necessary to combine elements from two competing hypotheses-the "retrieval effort" and the "retrieval success" hypotheses-about the role of this brain region in memory retrieval. The right prefrontal cortex is held to support the evaluation of the products of memory retrieval with respect to whether retrieved information represents a specific episode from the past. Thus, this region is engaged when retrieval is "successful" regardless of whether the retrieved information is accepted or rejected as bel onging to such an episode.

\section{ACKNOWLEDGMENTS}

M.D.R. is supported by a program grant and research leave fellowship from the Wellcome Trust. P.C.F., C.D.F., R.S.J .F., and R.J .D. are supported by the Wellcome Trust. K.A. is supported by a project grant from the Biotechnology and Biological Sciences Research Council of the United Kingdom.

\section{REFERENCES}

Allan, K., and Rugg, M. D. 1997. An event-related potential study of explicit memory on tests of word-stem cued recall and recognition memory. Neuropsychologia 35:387-397.

Allan, K., Wilding, E. L., and Rugg, M. D. 1998. Electrophysiological evidence for dissociable processes contributing to recollection. Acta Psychol . 98:231-252.

Buckner, R. L., Petersen, S. E., Ojemann, J . G., Miezin, F. M., Squire, L. R., and Raichle, M. E. 1995. Functional anatomical studies of 
explicit and implicit memory retrieval tasks. J. Neurosci. 15: 12-29.

Buckner, R. L., and Petersen, S. E. 1996. What does neuroimaging tell us about the role of prefrontal cortex in memory retrieval? Semin. Neurosci. 8:47-55.

Buckner, R. L., Raichle, M., Miezin, F. M., and Petersen, S. E. 1996. Functional anatomic studies of memory retrieval for auditory words and visual pictures. J . Neurosci. 16:6219-6235.

Buckner, R. L., Koutsaal, W., Schacter, D. L., Wagner, A. D., and Rosen, B. R. 1998a. Functional-anatomic study of episodic memory using fMRI. I. Retrieval effort versus retrieval success. Neurol mage 7:151-162.

Buckner, R. L., Koutsaal, W., Schacter, D. L., Dale, A. M., Rotte, M., and Rosen, B. R. 1998b. Functional-anatomic study of episodic memory using fMRI . II. Selective averaging of event-related fMRI trials to test the retrieval success hypothesis. Neurol mage 7:163175.

Cabeza, R., Kapur, S., Craik, F. I. M., Mcl ntosh, A. R., Houle, S., and Tulving, E. 1997. Functional neuroanatomy of recall and recognition: A PET study of episodic memory. J . Cognit. Neurosci. 9: 254-265.

Dolan, R. J ., and Fletcher, P. C. 1997. Dissociating prefrontal and hippocampal function in episodic memory encoding. Nature 388: 582-585.

Fletcher, P. C., Frith, C. D., Grasby, P. M., Shallice, T., Frackowiak, R. S. J ., and Dolan, R. J . 1995a. Brain systems for encoding and retrieval of auditory-verbal memory. An in vivo study in humans. Brain 118:401-416.

Fletcher, P. C., Frith, C. D., Baker, S. C., Shallice, T., Frackowiak, R. S. J ., and Dolan, R. J. 1995b. The mind's eye-Precuneus activation in memory-related imagery. Neurol mage 2:195-200.

Fletcher, P. C., Frith, C. D., and Rugg, M. D. 1997. Functional neuroanatomy of episodic memory. Trends Neurosci .20:213-218.

Francis, W. N., and Kucera, H. 1982. Computational Analysis of Present Day American Engl ish. Brown Univ. Press, Providence.

Friston, K. J ., Holmes, A. P., Worsley, K. J ., Poline, J -P., Frith, C. D., and Frackowiak, R. S. J. 1995. Statistical parametric maps in functional brain imaging: A general linear approach. Hum. Brain Mapping 2:189-210.

Kapur, S., Craik, F. I. M., J ones, C., Brown, G. M., Houle, S., and Tulving, E. 1995. Functional role of the prefrontal cortex in retrieval of memories-A PET study. NeuroReport 14:1880-1884.

Koriat, A., and Goldsmith, M. 1996. Monitoring and control processes in the strategic regulation of memory accuracy. Psychol. Rev.103: 490-517.
Nyberg, L., Tulving, E., Habib, R., Nilsson, L-G., Kapur, S., Houle, S., Cabeza, R., and Mclntosh, A. R. 1995. Functional brain maps of retrieval mode and recovery of episodic information. NeuroReport 7:249-252.

Rugg, M. D., Fletcher, P. C., Frith, C. D., Frackowick, R. S. J ., and Dolan, R. J . 1996. Differential activation of the prefrontal cortex in successful and unsuccessful memory retrieval. Brain 119:20732083.

Rugg, M. D., Fletcher, P. C., Frith, C. D., Frackowick, R. S. J ., and Dolan, R. J. 1997. Brain regions supporting intentional and incidental memory: A PET study. NeuroReport 8:1283-1287.

Schacter, D. L., Alpert, N. M., Savage, C. R., Rauch, S. L., and Albert, M. S. 1996a. Conscious recollection and the human hippocampal formation: Evidence from positron emission tomography. Proc. Natl. Acad. Sci. USA 93:321-325.

Schacter, D. L., Curran, T., Galluccio, L., Milberg, W. P., and Bates, J . F. 1996b. False recognition and the frontal lobe. Neuropsychologia 34:793-808.

Schacter, D. L., Reiman, E., Curran, T., Yung, L. S., Bandy, D., McDermott, K., and Roediger, H. L. 1996c. Neuroanatomical correlates of veridical and illusory recognition memory: Evidence from positron emission tomography. Neuron 17:267-274.

Shallice, T. 1988. From Neuropsychology to Mental Structure Cambridge Univ. Press, Cambridge, UK.

Silbersweig, D. A., Stern, E., Frith, C. D., Cahill, C., Schnorr, L., Grootoonk, S., Spinks, T., Clark, J ., Frackowiak, R. S. J ., and J ones, T. 1993. Detection of 30 second cognitive activations in single subjects with positron emission tomography: A new low-dose $\mathrm{H}_{2}{ }^{15} \mathrm{O}$ regional cerebral blood flow three dimensional imaging technique. J . Cereb. Blood Flow Metab. 13:617-629.

Squire, L. R. 1992. Memory and the hippocampus-A synthesis of findings from rats, monkeys, and humans. Psychol. Rev. 99: 195-231.

Squire, L. R., Ojemann, J . G., Miezin, F. M., Petersen, S. E., Videen, T. O., and Raichle, M. E. 1992. Activation of the hippocampus in normal humans: A functional anatomical study of memory. Proc. Natl. Acad. Sci. USA 89:1837-1841.

Talairach, J ., and Tournoux, P. 1988. Co-planar Stereotaxic Atlas of theHuman Brain. Thieme, Stuttgart.

Tulving, E., Kapur, S., Markowitsch, H. J ., Craik, F. I. M., Habib, R., and Houle, S. 1994. Neuroanatomical correlates of retrieval in episodic memory: Auditory sentence recognition. Proc. Natl. Acad. Sci. USA 91:2012-2015.

Wilding, E. L., and Rugg, M. D. 1996. An event-related potential study of recognition memory with and without retrieval of source. Brain 119:889-905. 